\title{
Norois
}

Environnement, aménagement, société

$245 \mid 2017$

Adapter les territoires aux changements climatiques : transition urbanistique et aménagement de l'espace

\section{Anticiper les impacts du changement climatique dans un milieu naturel soumis à de fortes contraintes anthropiques : l'estuaire de la Loire}

Anticipate climate change impacts in an estuarine environment under high anthropogenic pressure: the Loire estuary

Mathieu Le Dez, Jérôme Sawtschuk, Frédéric Bioret, Pierre Le Hir et Régis Walther

\section{OpenEdition}

\section{Journals}

Édition électronique

URL : https://journals.openedition.org/norois/6206

DOI : $10.4000 /$ norois.6206

ISBN : 78-2-7535-7465-6

ISSN : $1760-8546$

Éditeur

Presses universitaires de Rennes

Édition imprimée

Date de publication : 31 décembre 2017

Pagination : 15-28

ISBN : 978-2-7535-7456-4

ISSN : 0029-182X

\section{Référence électronique}

Mathieu Le Dez, Jérôme Sawtschuk, Frédéric Bioret, Pierre Le Hir et Régis Walther, « Anticiper les impacts du changement climatique dans un milieu naturel soumis à de fortes contraintes anthropiques : l'estuaire de la Loire », Norois [En ligne], 245 | 2017, mis en ligne le 31 décembre 2019, consulté le 13 janvier 2022. URL : http://journals.openedition.org/norois/6206 ; DOI : https://doi.org/ $10.4000 /$ norois. 6206 


Presses
Universitaires
de Rennes

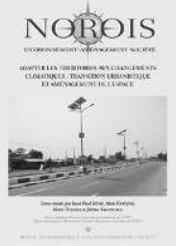

\title{
Anticiper les impacts du changement climatique dans un milieu naturel soumis à de fortes contraintes anthropiques : l'estuaire de la Loire
}

\author{
Anticipate Climate Change Impacts in an Estuarine Environment \\ under High Anthropogenic Pressure: The Loire Estuary
}

\author{
Mathieu Le Deza , Jérôme SawtschuK , Frédéric Bioneta \\ Pierre Le Hir ${ }^{b}$ et Régis WALther ${ }^{c}$
}

\footnotetext{
a EA 7462, Géoarchitecture, université de Bretagne Occidentale, Brest. (mathieu.ledez29@gmail.com) (jerome.sawtschuk@univ-brest.fr)

' IFREMER, Laboratoire DYNECO/PHYSED, Plouzané.

cARTELIA Eau et Environnement, Grenoble.
}

Résumé : Dans les prochaines décennies, les effets du changement climatique devraient modifier le fonctionnement hydro-sédimentaire des grands estuaires français. Deux projets de recherche s'intéressent depuis 2011 aux changements environnementaux se produisant dans l'estuaire de la Loire. Ces travaux ont notamment pour objectif d'évaluer les conséquences des évolutions du fonctionnement hydro-sédimentaire sur les milieux naturels des zones latérales inondables et d'évaluer les effets sur les activités humaines et leurs prises en considération par les usagers de ces espaces. Après une présentation globale du territoire d'étude correspondant aux complexes prairiaux de la rive nord de l'estuaire de la Loire, les impacts attendus du changement climatique dans les prochaines décennies sont présentés et confrontés aux enjeux socio-économiques de ces territoires.

L'aménagement industrialo-portuaire de l'estuaire de la Loire a provoqué des changements importants sur la rive nord de l'estuaire de la Loire lors des trois dernières décennies. Ces modifications passées comparées aux résultats de simulation du modèle hydrosédimentaire mettent en évidence d'importantes évolutions à prévoir dans les prochaines décennies en lien avec le changement climatique : submersions plus importantes de la plaine alluviale, progression de la salinité vers l'amont, développement du bouchon vaseux. Les usages de ce territoire, plus ou moins liés au fleuve (activités industrialo-portuaires, agriculture, protection de la nature...), subiront les conséquences de ces changements, et nécessiteront probablement des adaptations.

L'étude croisée des dynamiques écologiques, hydro- sédimentaires et socio-économiques constitue une approche innovante pour appréhender la réponse d'un socio écosystème aux changements environnementaux. Les données produites dans le cadre de tels travaux de recherche peuvent permettre d'accompagner les gestionnaires du territoire dans la mise en œuvre d'un projet de transition pour minimiser et accompagner les effets du changement climatique.

\footnotetext{
Abstract: Within the next decades, the effects of climate change are expected to alter the hydro-sedimentary functioning of large French estuaries. Since 2011, two research projects have been implemented about environmental changes in the Loire estuary to assess their consequences on the natural environments of flood zones and their impacts on human activities. After an overall presentation of the study area, a wetland complex located on the north shore of the Loire estuary, the expected impacts of climate change in the coming decades are presented.

The industrial-port development of the estuary has caused considerable changes on the north shore of the Loire estuary during the last three decades. These past changes, compared to the results of the simulation of the hydro-sedimentary model, highlight important evolutions to
} 
be expected in the coming decades in relation to climate change: greater submersion of the allwvial plain, salinity upstream, and increase of sediment concentration. Industrial-port activities, agriculture, protection of nature and other activities, will suffer the consequences of these changes, and will probably require adaptations.

The cross-study of ecological, hydro-sedimentary and socio-economic dynamics developed in this study consists in an innovative approach to understanding the response of a socio-ecosystem to environmental changes. The data produced in the course of such research, if shared with local stakeholders, can contribute to in the implementation of a transition project to minimize and accompany the effects of climate changes.

Mots clés : Changement climatique - Estuaire - modélisation - biodiversité végétale - impacts environnementaux et socio-économiques - transition écologique - aménagement fluvial

Keywords: Climate change - Estuary - modeling - plant biodiversity - environmental and socio-economical impacts - ecological transition - hydraulic engineering

\section{INTRODUCTION}

Les grands estuaires français, par leur position d'interface entre les eaux continentales et les eaux marines, ainsi que par la faible altitude de leur plaine alluviale, font partie des zones littorales qui devraient subir de manière importante les conséquences du changement climatique (Day et al., 2008). En effet, selon les prévisions (GIEC, 2007; Robins et al., 2016) l'influence du changement climatique sur les estuaires se manifestera surtout au niveau hydro-sédimentaire avec une modification du niveau marin et du régime des tempêtes en aval, et une évolution des apports liquides et solides en amont.

Afin d'anticiper les impacts potentiels du changement climatique sur ces espaces, le projet de recherche C3E2 (Conséquences du Changement Climatique sur l'Écogéomorphologie des Estuaires) a été mené entre 2011 et 2014, dans le cadre du programme Gestion et Impacts du Changement Climatique mené par le ministère de l'Écologie, du Développement Durable et de l'Énergie. Les objectifs de ce projet étaient de préciser les conséquences du changement climatique sur les estuaires en termes de morphologie et de couverture sédimentaire, puis d'en évaluer les conséquences sur les milieux naturels des zones latérales inondables. Pour réaliser ce projet, l'estuaire de la Loire a été utilisé comme site d'étude (Le Hir, 2014).

S’inscrivant dans la continuité de C3E2, le projet de recherche ICEPEL (Impacts des Changements Environnementaux sur les complexes Prairiaux de l'Estuaire de la Loire), lancé en 2015 dans le cadre du programme « quels littoraux pour demain » de la Fondation de France, s’intéresse aux impacts de ces changements sur les activités humaines et leurs prises en considération par les usagers de ces espaces.

Cet article s'appuie sur les résultats de ces deux projets de recherche et a pour objectif d'envisager les conséquences des changements environnementaux en cours ou attendus sur l'aménagement de l'estuaire de la Loire pour répondre à plusieurs questions :

- les modélisations des dynamiques végétales et hydro-sédimentaires permettent-elles d'anticiper les effets du changement climatique sur les activités socio-économiques d'un territoire?

- Les connaissances scientifiques acquises sur un territoire peuvent-elles contribuer à une prise de conscience nécessaire à une évolution des politiques d'aménagement et d'urbanisme?

\section{Présentation du site d’étude}

\section{Une interface entre l'océan et le continent}

Dans le cadre du projet C3E2, l'estuaire de la Loire (figure 1 - planche I) a été choisi comme site pilote, car il possède les principales caractéristiques de fonctionnement hydro-sédimentaire des grands estuaires français :

- l'exutoire d'un large bassin versant permettant des apports importants d'eau douce et de sédiments de l'amont;

- une large ouverture sur l'océan couplée à une importante propagation de la marée dans l'estuaire, apportant l'eau salée et les sédiments de l'aval;

- la présence d'un bouchon vaseux, phénomène typique des grands estuaires, correspondant à une 
importante masse de matières en suspension se concentrant à la rencontre des eaux douces et des eaux salées;

- une plaine alluviale de faible altitude subissant les débordements périodiques du fleuve.

\section{Un territoire anthropisé, en transition}

Souvent présentée comme le dernier fleuve sauvage d'Europe, la Loire a subi d'importants aménagements par l'homme, en particulier dans sa partie estuarienne.

Dès le Moyen Âge, les marais de la plaine inondable du fleuve, régulièrement submergés, ont été progressivement aménagés avec la création de canaux et de douves gérés par des vannes (Estuarium, 1997 ; Desprès, 2009). Ce vaste réseau hydraulique a permis de réguler les inondations de ces zones humides et rendu possible leur utilisation pour l'agriculture (GIP Loire estuaire, 2011a).

Puis, la révolution industrielle a conduit au développement de la vocation industrialo-portuaire de l'estuaire à partir du XVIII ${ }^{\mathrm{e}}$ siècle. Plusieurs phases d'aménagements du fleuve ont été menées pour faciliter le transport maritime et permettre la remontée des navires jusqu'au port de Nantes (création d'endiguements, approfondissement du lit du fleuve...) (Fleury, 1997; Demaure, 1979, Ottmann, 1987, Verger, 2005). Parallèlement à ces travaux, l'implantation des sites industrialo-portuaires a entraîné une importante artificialisation et urbanisation des secteurs amont et aval de l'estuaire : création de remblais, construction des infrastructures portuaires et industrielles, développement urbain des communes environnantes, etc. (Cabanne, 1985; Mareï et Tourret, 2009; INSEE, 2008). Ces aménagements ont provoqué une transformation profonde de la morphologie du fleuve avec un rattachement progressif des îles au rivage lié au comblement d'anciens bras et à la réduction de la largeur du fleuve (Verger, 2005). Ils ont aussi modifié le fonctionnement hydro-sédimentaire : remontée de la salinité vers l'amont, développement du bouchon vaseux, abaissement des niveaux de basses eaux, etc. (CSEEL, 1984; Migniot et al, 1997).

Notre territoire d'étude principal se situe sur la rive nord de l'estuaire de la Loire entre SaintNazaire et Nantes (figure 1 - planche I). C'est un secteur peu urbanisé qui se caractérise par la présence de grands espaces de prairies inondables. Cette rive a subi d'importantes modifications lors des dernières décennies liées à l'aménagement de l'estuaire (atterrissement progressif des îles de Loire, remblais). Ce secteur a été retenu car il bénéficie de données cartographiques précises sur la dynamique de la végétation depuis 1982 (Dupont et al., 1983; Sawtschuk et Bioret, 2012; Le Dez et al., 2017) et d'un modèle hydro-sédimentaire fin (figure 2 - planche I). Il a la particularité d'être situé au centre du pôle métropolitain Nantes SaintNazaire qui regroupe 61 communes et 6 intercommunalités et plus de 830000 habitants (87\% dans les agglomérations de Nantes et Saint-Nazaire). Le secteur étudié concerne les communes situées entre ces deux villes et concerne quatre intercommunalités : Nantes Métropole, CARENE, Loire et Sillon et Cœur d'Estuaire.

Le Schéma de cohérence territoriale (SCoT) Nantes Saint-Nazaire adopté en 2007, et le suivant qui débute en 2017, montrent que le projet de ce territoire s'inscrit dans une démarche de transition écologique. Ces documents d'urbanisme mettent en avant le besoin d'agir pour la réduction de la production des gaz à effet de serre et la maîtrise des dérèglements climatiques de la planète ainsi que pour la protection de l'environnement encore préservé tel que celui des zones humides de l'estuaire.

\section{Des milieux naturels associés au fleuve}

Entre les deux unités urbaines de Nantes et de Saint-Nazaire, le secteur non-urbanisé de la plaine alluviale de la Loire constitue "l'écharpe verte ${ }^{1}$ " de l'estuaire (Mauvais et Goarnisson, 1999). Cet espace naturel, bien qu'ayant été fortement modifié par l'homme au cours des derniers siècles (assèchements, remblais...), forme encore aujourd'hui une zone humide d'importance majeure (Leiderman et Mermet, 1991). Submergé périodiquement par les eaux du fleuve, ce territoire est composé d'une mosaïque de prairies et de roselières caractérisées par leur régime d'inondation et leur salinité. Ces espaces abritent une végétation et une faune reconnues d'intérêt patrimonial, et sont intégrés dans un site Natura 2000 (figure 1 - planche I). La faible altitude de la plaine alluviale (plus de la moitié de la

1. Dénomination utilisée dans les documents d'urbanisme (Directive Territoriale d'Aménagement) 
surface se situe à une altitude inférieure à la ligne de rive (2,70 mètres IGN69 - GIP Loire estuaire, 2012) permet des débordements pouvant recouvrir plus de 8000 hectares (GIP Loire estuaire, 2005) et rend ce territoire potentiellement sensible aux effets du changement climatique (élévation du niveau de la mer, modification du régime des tempêtes). Les dynamiques de ces milieux humides sont liées en grande partie aux activités agricoles en lien avec l'élevage (pâturage, fauche). Sur notre secteur d'étude, des changements majeurs de végétation se sont produits depuis une trentaine d'années au niveau des bords de Loire, notamment dans la partie située entre Cordemais et Donges (Le Dez et al., 2017).

\section{Des usages dépendants du fleuve}

Une grande partie des usages qui existent sur ce territoire dépendent plus ou moins fortement du fleuve.

- L'agriculture : principale activité sur la plaine alluviale. Les agriculteurs tirent profit des prairies inondables pour y faire pâturer leur bétail et y produire un foin de qualité (Chambre d'agriculture de Loire Atlantique, GIP Loire estuaire et DDTM 44, 2013).

- La chasse au gibier d'eau : la situation privilégiée de l'estuaire de la Loire comme axe stratégique pour la migration d'un nombre important d'espèces d'oiseaux chassables a conduit au développement de la chasse au gibier d'eau (GIP Loire estuaire, 2006a).

- La protection de la nature : zone humide d'importance majeure, ce territoire présente de nombreux enjeux de conservation de la biodiversité (faune, flore, habitats). Plusieurs associations naturalistes agissent pour la préservation de ces espaces (Association pour la Connaissance et la Recherche Ornithologique en Loire et Atlantique, Ligue pour la Protection des Oiseaux...), ainsi que le Conservatoire de l'espace littoral et des rivages lacustres qui a acquis plusieurs centaines d'hectares de marais gérés par le Conseil départemental de Loire-Atlantique et l'Office national de la chasse et de la faune sauvage.

- Zones habitées : les points plus élevés, situés sur les pourtours de la plaine inondable, ont permis l'implantation d'habitations qui peuvent subir des inondations lors des submersions les plus importantes de la Loire. Les zones d'habitation les plus anciennes correspondent principalement à d'anciens ports aujourd'hui en partie déconnectés du fleuve, comme à Lavau-sur-Loire ou à des exploitations agricoles. L'urbanisation des dernières décennies s'explique davantage par le développement de nouvelles activités industrielles (Donges, Cordemais et terminaux de Montoir) et par le phénomène de périurbanisation de Nantes et de Saint-Nazaire, comme sur le territoire de la commune de Couëron.

- Activités industrialo-portuaires : la circulation des bateaux dépend des profondeurs d'eau dans le fleuve, notamment régulées par les dragages du chenal de navigation (GIP Loire Estuaire, 2011b). Certaines industries nécessitent des prélèvements d'eau dans la Loire comme la centrale thermique de Cordemais ou le terminal méthanier de Montoir de Bretagne) (GIP Loire Estuaire, 2011c).

\section{MÉTHOde POUR L'ÉTUde de L'IMPACT DU CHANGEMENT CLIMA- TIQUE SUR L'ESTUAIRE DE LA LOIRE}

\section{Évolution hydro-sédimentaire}

L'évaluation des conséquences du changement climatique a été réalisée à l'aide d'une modélisation numérique (figure 2 - planche I). Le modèle utilisé a été développé pour le GIP Loire estuaire par le bureau d'études Artélia à l'aide de l'outil Telemac-3D (Walther et David, 2010; Walther et al., 2012; Le Hir, 2014). Il permet de simuler le fonctionnement de l'estuaire en y intégrant les principaux paramètres hydro-sédimentaires agissant sur ce territoire (niveaux d'eau, dépôts de vase, salinité...).

Ce modèle a été utilisé pour réaliser des projections à long terme (jusqu'en 2040), en y associant les effets attendus du changement climatique sur l'estuaire de la Loire. Ces derniers ont été évalués sur la base d'une synthèse de travaux scientifiques (Day et al., 2008) qui a permis de retenir deux principaux phénomènes susceptibles d'impacter le fonctionnement hydro-sédimentaire de l'estuaire :

- L'élévation du niveau de la mer, en intégrant un niveau moyen de $+0,34$ m d'ici 2040 (correspond à l'hypothèse extrême du GIEC, soit + 1 m en 2100).

- Les modifications de la pluviosité et de l'évapotranspiration sur le territoire du bassin versant de la Loire qui provoqueraient une réduction du 
débit (selon les résultats du projet de recherche HYDROQUAL-Impact du Changement Climatique sur l'hydrosystème Loire (Moatar et al., 2010), pour un scénario du GIEC entraînant une réduction maximale des débits en 2040).

Sur la base de ces hypothèses, quatre scénarios sont étudiés :

- Scénario sans changement climatique

- Scénario avec élévation du niveau de la mer (+1 m en 2100; scenario extrême GIEC)

- Scénario avec modification de l'hydrologie amont (Forçage issu du projet HYDROQUAL : diminution des débits et diminution des apports solides)

- Scénario combiné avec élévation du niveau de la mer et modification de l'hydrologie amont

Ils sont présentés en détail dans le rapport de recherche du projet de recherche C3E2 (Le Hir, 2014).

\section{Dynamique de la végétation}

Afin d'évaluer la réponse de la végétation aux modifications des paramètres hydro-sédimentaires (niveaux d'eau, dépôts de vase, salinité...), les dynamiques passées des milieux naturels de la plaine inondable ont été étudiées à l'aide d'un système d'information géographique et du modèle des matrices de transitions (Usher, 1992; Gourmelon et al., 2003). Les aménagements de l'estuaire de la Loire réalisés au cours du $\mathrm{xx}^{\mathrm{e}}$ siècle ont modifié de façon importante le fonctionnement hydro-sédimentaire de l'estuaire (remontée vers l'amont de la salinité, développement du bouchon vaseux, accrétions sédimentaires latérales...) (CSEEL, 1984; Migniot et al., 1997). L'étude des dynamiques passées de la végétation a été réalisée pour émettre des hypothèses sur les conséquences des modifications du fonctionnement hydro-sédimentaire attendues avec le changement climatique.

Les trois cartes de végétation réalisées en 1982 (Dupont et al., 1983), 2002 (Ouest Aménagement, 2003) et 2014 (Le Dez et al., 2017), ont permis d'effectuer une analyse diachronique portant sur les trois dernières décennies (figure 3 - planche II). Sur la base des connaissances en écologie végétale, chaque type de végétation a été caractérisé en fonction de son degré d'exposition au sel et aux inondations, principaux facteurs écologiques qui agissent sur ces marais. Le diagramme de la figure 4 (planche II) permet une représentation des groupements de végétation en fonction de ces deux facteurs (gradient de salinité et gradient d'humidité).

\section{ConséquencES DU CHANGEMENT CLIMATIQUE SUR LES MILIEUX NATURELS}

Les résultats présentés ci-après sont issus de la modélisation intégrant le scénario combiné (élévation du niveau de la mer + changement de l'hydrologie en amont) jugé comme étant l'un des plus probables. Les conséquences sur les milieux naturels sont envisagées sur la base des connaissances acquises sur l'écologie des groupements de végétation (exigences écologiques, dynamiques) et mises en regard des résultats du modèle hydro-sédimentaire.

\section{Une pénétration accrue des eaux salées dans l'estuaire favorable au développe- ment des végétations halophiles}

Les résultats de la modélisation font apparaître d'ici 2040, une progression de la salinité vers l'amont d'environ $4 \mathrm{~km}$ par rapport à la situation actuelle. Ce phénomène serait principalement favorisé par la réduction des débits en lien avec des pluviosités plus faibles attendues sur le bassin versant de la Loire. Ce mécanisme serait accentué par l'élévation du niveau de la mer qui permettrait une légère augmentation de l'intrusion saline.

Comme présenté en introduction, la remontée du système halin a déjà été observée au cours du $\mathrm{xx}^{\mathrm{e}}$ siècle, suite aux travaux d'aménagement de l'estuaire pour la navigation, qui ont favorisé la progression de la marée vers l'amont (CSEEL, 1984 ; Migniot et al., 1997). L'analyse des cartographies de végétation présentées dans la figure 3 (planche II) permet de constater les conséquences de ce phénomène sur les milieux naturels de la plaine inondable. Ainsi, entre 1982 et 2014, on peut observer le développement des végétations halophiles (végétations des milieux salés ou saumâtres) et la réduction de la surface des végétations des milieux doux. Cette transformation s'observe principalement dans la partie aval avec le remplacement de la petite roselière douce (93,6 ha en 1982 à 6 ha en 2014), par la petite roselière saumâtre $(4,8$ ha en 1982 à 
92,3 ha en 2014) ou la roselière douce à saumâtre. De même, durant cette période, certains secteurs de prairies ont été colonisés par des végétations de prés salés, autrefois localisées plus en aval de l'estuaire (Dupont et al., 1983).

\section{Un développement et une remontée vers l'amont du bouchon vaseux favori- sant les atterrissements latéraux}

Le modèle fait également apparaître que le changement climatique entraînerait un étalement vers l'amont du bouchon vaseux, principalement causé par les débits plus faibles. Cette configuration pourrait favoriser le piégeage des sédiments.

De la même manière que précédemment, ce phénomène a déjà été observé au cours du $\mathrm{xx}^{\mathrm{e}}$ siècle, suite aux travaux d'aménagement de l'estuaire. L'accumulation des sédiments sur les vasières a notamment favorisé le mécanisme d'accrétion sédimentaire qui a conduit à la progression de la ligne de rive de plusieurs dizaines de mètres en quelques années (Gras, 1981). Cette accrétion sédimentaire se poursuivrait dans les prochaines décennies, indépendamment des changements climatiques car certaines zones basses ne sont pas encore arrivées à l'équilibre sédimentaire provoqué par les aménagements de l'estuaire (chenal de navigation et remblais). L'analyse des cartes de végétation montre que ce mécanisme a permis la progression de la végétation vers la Loire, sur des zones qui étaient à l'origine non végétalisées. L'examen détaillé de ce phénomène à travers les cartes de la figure 3 (planche II) met en évidence, dans un premier temps, une colonisation par la petite roselière saumâtre, remplacée dans un second temps par la roselière douce à saumâtre. Finalement, le rehaussement progressif et la stabilisation des vasières, permet leur exploitation par l'agriculture (fauche ou pâturage) conduisant ainsi au développement des végétations prairiales (prairie ou pré salé).

D'autre part, lors des submersions de la plaine alluviale par les eaux chargées en sédiment du bouchon vaseux, des dépôts importants de vase ont pu être observés sur les prairies (Chambre d'agriculture de Loire Atlantique, GIP Loire estuaire et DDTM 44, 2013). Souvent associé à des dépôts de sel, ce phénomène provoque l'asphyxie de la végéta- tion en place, puis la recolonisation par des espèces annuelles pionnières nitro-halophiles (groupement à Atriplex hastata) (Sawtschuk et Bioret, 2012).

\section{Une augmentation des submersions de la plaine alluviale favorisant le développement des roselières}

Les résultats de la modélisation mettent en évidence des inondations plus courantes de la plaine alluviale à l'horizon 2040, en lien direct avec l'élévation du niveau de la mer. Ainsi, les temps de dépassements d'un seuil de $20 \mathrm{~cm}$ d'eau seraient doublés en moyenne d'ici 30 ans. Ce phénomène conduirait à accentuer l'exposition des milieux naturels de la plaine alluviale au sel et aux sédiments du bouchon vaseux.

L'augmentation des submersions n'a pas été observée dans le passé et il n'est donc pas possible d'en analyser les conséquences sur la végétation par l'analyse diachronique. En revanche, la connaissance des exigences écologiques des différents types de végétation, synthétisée sur la figure 4 (planche II), permet d'émettre certaines hypothèses. On peut ainsi penser que l'augmentation des temps d'inondation favoriserait le développement des hélophytes (roselières) mieux adaptées aux immersions, en particulier dans les parties les plus basses des marais. De plus, l'exposition prolongée au sel et aux dépôts de vase accentuerait les transformations de la végétation évoquées précédemment (développement des végétations halophiles au détriment de celles des milieux doux, apparitions des végétations pionnières).

\section{Discussion}

\section{La rive nord de l'estuaire de la Loire : un territoire en mouvement}

Les projets de recherches et les études développés sur les changements environnementaux dans l'estuaire de la Loire contribuent à une meilleure appréhension des phénomènes physique, chimique, biologique et socio-économique en cours ou à venir dans ce territoire. Les dynamiques observées lors des trois dernières décennies au niveau des complexes prairiaux de la rive nord de l'estuaire résultent en grande partie des effets des aménagements industrialo-portuaires qui ont modifié le fonctionnement 
hydro-sédimentaire du fleuve. Les changements observés sur la végétation révèlent des modifications hydro-sédimentaires et de la salinité, phénomènes liés à des modifications anthropiques de la morphologie l'estuaire (creusement du chenal de navigation). Si certaines évolutions résultent d'une modification locale des usages des terrains par l'agriculture ou la chasse (Le Dez et al., 2017), les aménagements portuaires et industriels à l'échelle de l'estuaire ont provoqué artificiellement des dépôts sédimentaires et une progression de la salinité d'une telle ampleur que l'on pourrait caractériser certains secteurs des bords de Loire de nouveaux écosystèmes anthropiques (Saeijs, 1982; Hobbs et al., 2009). Parmi ces changements environnementaux, il est difficile d'identifier pour l'instant des conséquences avérées des changements climatiques. Les modélisations hydrosédimentaires réalisées selon différents scénarios montrent que ce territoire continuerait dans tous les cas à se modifier, notamment d'un point de vue sédimentaire, en réponse à l'aménagement portuaire, même sans changements climatiques (Le Hir, 2014).

La modélisation met cependant en évidence des impacts significatifs du changement climatique sur le fonctionnement hydro-sédimentaires à prévoir lors des prochaines décennies avec notamment une progression de la salinité vers l'amont et un développement et une remontée vers l'amont du bouchon vaseux. On peut donc anticiper que les changements de végétation observés depuis trente ans sur les complexes prairiaux situés à l'aval de l'estuaire se produiraient probablement plus en amont, à proximité de Nantes avec une arrivée d'espèces halophiles prévisible. En confrontant ces phénomènes aux enjeux socio-économiques de ces territoires il devient possible d'anticiper les effets du changement climatique sur le fonctionnement des activités humaines qui se déroulent sur les complexes prairiaux (tableau 1).

\section{Impacts prévisibles du changement climatique sur les activités humaines}

\section{Agriculture}

Principale activité menée sur la plaine inondable, l'agriculture serait probablement très impactée par ces changements environnementaux. L'augmentation des durées de submersion risque de limiter les périodes d'exploitation des prairies (diminution du temps de pâturage pour le bétail) et éventuellement contraindre les accès à certaines

\begin{tabular}{|c|c|c|c|c|c|}
\hline \multirow[b]{2}{*}{ Paramètres } & \multirow{2}{*}{$\begin{array}{c}\text { Effets attendus des } \\
\text { changements clima- } \\
\text { tiques }\end{array}$} & \multicolumn{4}{|c|}{ Impacts à prévoir sur les activités humaines } \\
\hline & & Agriculture & Chasse & $\begin{array}{c}\text { Protection de la } \\
\text { nature }\end{array}$ & Urbanisation \\
\hline \multirow{3}{*}{$\begin{array}{l}\text { Hydro- } \\
\text { sédimen- } \\
\text { taires }\end{array}$} & $\begin{array}{c}\text { Augmentations } \\
\text { durées et niveaux de } \\
\text { submersion }\end{array}$ & $\begin{array}{l}\text { Diminution périodes } \\
\text { d'exploitation des } \\
\text { prairies, difficultés } \\
\text { d'accès aux parcelles }\end{array}$ & Problèmes d'accès & $\begin{array}{c}\text { En faveur des zones } \\
\text { humides estuariennes }\end{array}$ & $\begin{array}{l}\text { Habitations inon- } \\
\text { dées, besoin de } \\
\text { renforcer les ouvrages } \\
\text { de protection }\end{array}$ \\
\hline & $\begin{array}{l}\text { Augmentation de la } \\
\text { salinité de l'estuaire }\end{array}$ & $\begin{array}{l}\text { Prairies « grillées » } \\
\text { par le sel, problème } \\
\text { abreuvement du } \\
\text { bétail }\end{array}$ & & $\begin{array}{c}\text { Recul espèces proté- } \\
\text { gées sensibles au sel } \\
\text { (p. ex. Angélique des } \\
\text { estuaires) }\end{array}$ & $\begin{array}{l}\text { Problème alimenta- } \\
\text { tion en eau potable }\end{array}$ \\
\hline & $\begin{array}{l}\text { Développement du } \\
\text { bouchon vaseux vers } \\
\text { l'amont }\end{array}$ & $\begin{array}{l}\text { Prairies recouvertes } \\
\text { par la vase lors des } \\
\text { débordements }\end{array}$ & & $\begin{array}{c}\text { Diminution de l'oxy- } \\
\text { gène de l'eau : impact } \\
\text { sur des espèces } \\
\text { patrimoniales (p. ex. } \\
\text { saumon) }\end{array}$ & $\begin{array}{l}\text { Perturbation prélève- } \\
\text { ment d'eau centrale } \\
\text { thermique }\end{array}$ \\
\hline \multirow{2}{*}{ Végétation } & $\begin{array}{l}\text { Progression des végé- } \\
\text { tations halophiles }\end{array}$ & $\begin{array}{l}\text { Modification valeur } \\
\text { fourragère, produc- } \\
\text { tivité }\end{array}$ & $\begin{array}{l}\text { Modification de } \\
\text { la faune }\end{array}$ & $\begin{array}{c}\text { Favorable aux végé- } \\
\text { tations ne pouvant } \\
\text { s'exprimer à l'aval }\end{array}$ & \\
\hline & $\begin{array}{l}\text { Progression des } \\
\quad \text { roselières }\end{array}$ & $\begin{array}{l}\text { Diminution des sur- } \\
\text { faces de prairies }\end{array}$ & Sanglier favorisé & $\begin{array}{c}\text { Effets significatifs } \\
\text { sur l'avifaune (p. ex. } \\
\text { Phragmite aquatique) }\end{array}$ & \\
\hline
\end{tabular}

Tableau 1 : Effets attendus des changements climatiques sur les activités humaines se déroulant sur les bords de Loire Expected effects of climate change on human activities taking place on the banks of the Loire River 
parcelles. La sous-exploitation, voire l'abandon des pratiques agricoles (fauche, pâturage), tendrait à favoriser la dynamique naturelle de la végétation et le développement des roselières, ce qui conduirait finalement à réduire les surfaces de prairies utilisables pour l'agriculture.

Les changements de la végétation (déjà observés dans le passé : développement des espèces de prés salés, apparition de végétations pionnières...) impliquent potentiellement des modifications de la qualité agronomique des prairies (valeur fourragère, productivité...) et peuvent donc avoir un impact sur la rentabilité de celles-ci. Quelques agriculteurs ont constaté que, dans certaines situations, le recouvrement des prairies par les eaux salées pouvait conduire à « griller » la végétation, et ainsi provoquer la perte temporaire de surfaces de prairies (Chambre d'agriculture de Loire Atlantique, GIP Loire estuaire et DDTM 44, 2013).

L'augmentation de la salinité dans les mares utilisées pour l'abreuvement du bétail, oblige également à apporter de l'eau douce pour les animaux, ce qui entraîne une contrainte supplémentaire dans l'exploitation de ces espaces (Chambre d'agriculture de Loire Atlantique, GIP Loire estuaire et DDTM 44, 2013).

\section{Chasse}

De la même manière que pour l'agriculture, l'augmentation des périodes de submersion risque de réduire l'accès aux mares de chasses et de modifier la pratique de cette activité. Il est par exemple possible que ces submersions favorisent à terme la présence de zones humides favorables au gibier d'eau.

\section{Protection de la nature}

Les modifications du fonctionnement hydro-sédimentaire engendrent des changements des conditions de vie des espèces vivant dans l'estuaire. Dans le fleuve, le développement du bouchon vaseux vers l'amont en étiage peut entraîner en été une augmentation des hypoxies voire des crises d'anoxie, menaçant les poissons, dont certaines espèces à forte valeur patrimoniale comme le saumon (GIP Loire Estuaire, 2006b). Sur la plaine alluviale, les changements de végétation et l'évolution de la salinité peuvent menacer certaines espèces rares par la suppression ou la réduction des milieux favorables à leur développement. L'Angélique des estuaires, espèce végétale endémique des estuaires atlantiques français, pourrait ainsi être menacée par les effets du changement climatique qui seraient susceptibles de compromettre sa conservation à long terme (Magnanon et al., 1998, Cianfaglione et Bioret, 2017).

Il est également possible de penser que certaines conséquences auront des impacts positifs sur le milieu naturel. L'augmentation des submersions permettrait potentiellement un maintien des fonctionnalités écologiques estuariennes, qu'une sédimentation continue de la plaine alluviale ferait disparaître (réduction des zones intertidales...). La progression de la salinité pourrait permettre la remontée de groupements de végétation halophiles, ne pouvant pas (ou peu) s'exprimer actuellement sur les secteurs très aménagés de l'aval (complexes industrialo-portuaires de Saint-Nazaire, Montoir-deBretagne, Donges).

\section{Zones habitées}

Déjà touchées lors d'événements importants comme la tempête Xynthia en 2011 , certaines habitations risquent d'être plus souvent soumises aux inondations avec des submersions plus importantes attendues sur la plaine alluviale. L'entretien ou le développement des étiers, des ouvrages hydrauliques et des levées de terre existants seront déterminants pour le maintien à long terme de certains secteurs habités.

\section{Industries/navigation}

Le bouchon vaseux a déjà contraint à limiter les prélèvements d'eau dans la Loire et conduit à des arrêts d'exploitation de la centrale thermique de Cordemais (GIP Loire Estuaire, 2006 b). Son développement dans l'avenir entraînerait probablement des perturbations similaires plus fréquentes.

\section{Prise en compte des modifications hydro-sédimentaires dans l'aménagement du territoire}

Face aux impacts des modifications du fonctionnement hydro-sédimentaire évoqués précédem- 
ment, des réflexions sont menées depuis plusieurs années pour tenter de limiter les effets négatifs de ces changements. Plusieurs projets, visant l'amélioration des conditions estuariennes, ont été envisagés (GIP Loire Estuaire, 2007 ; Guillemot, 2015) :

- un barrage d'estuaire;

- la réouverture de certains bras morts;

- la restauration de vasières.

Ces différents projets avaient pour principal objectif de réduire l'influence maritime de l'estuaire permettant notamment de limiter les entrées d'eaux salées et le développement du bouchon vaseux. Compte tenu des coûts élevés de mise en œuvre et de l'incertitude envers la pérennité de leur efficacité, aucun de ces projets n'a vu le jour actuellement.

\section{Les connaissances sur les dynamiques de l'estuaire comme outil d'aide à la décision pour l'aménagement}

Les résultats du projet de recherche C3E2 ont permis d'envisager les impacts du changement climatique sur le fonctionnement hydro-sédimentaire de l'estuaire de la Loire et les conséquences sur la végétation de la plaine alluviale à l'horizon 2040. Les connaissances acquises sur les activités humaines de ce territoire, à travers le programme ICEPEL, sont maintenant utilisées pour envisager les conséquences de ces changements sur les usages et leur prise en compte dans l'aménagement du territoire. Les connaissances scientifiques acquises sur le territoire de la rive nord de l'estuaire sont progressivement intégrées aux réflexions en cours sur l'aménagement de ce territoire. Les données cartographiques très précises sur la végétation, qui résultent d'inventaires de terrains répétés dans le temps permettent de donner des éléments « objectifs » sur des changements en cours des milieux. Ces données sont par exemple utilisées par le Conseil départemental de Loire-Atlantique pour la gestion des terrains du Conservatoire du littoral dont le plan de gestion vient d'être finalisé, ou par le GIP Loire Estuaire pour affiner la connaissance de la dynamique des roselières. D'après le GIP, les données de modélisation hydro-sédimentaire ont été quant à elles utilisées récemment pour justifier le projet de réserve naturelle nationale de l'estuaire de la Loire. Les connaissances sur les évolutions passées, en cours et à venir de ce territoire peuvent donc consti- tuer des outils d'aide à la décision qui contribuent à une évolution des politiques d'aménagement. L'ampleur des changements environnementaux, en cours, ou à venir au niveau de l'estuaire de la Loire met en évidence la nécessité de mettre en œuvre des suivis scientifiques rigoureux pour mieux appréhender les phénomènes complexes dans le temps et l'espace.

Des limites existent cependant à l'utilisation de ces données : celles-ci doivent être partagées et faire l'objet d'une part d'une médiation scientifique en direction du secteur associatif, et d'autre part d'une meilleure concertation dans leur acquisition et leur valorisation avec les laboratoires de recherche impliqués sur l'estuaire. Le GIP Loire Estuaire doit assurer un rôle central en ayant pour vocation de mettre en relation les acteurs de l'estuaire et en favorisant le partage des bases de données, et la réalisation de publications et conférences. Le syndicat Loire aval (Syloa), créé en 2015 pour porter le SAGE Estuaire de la Loire est aussi un organisme clé pour la valorisation de ces connaissances et leur prise en compte dans les documents d'urbanisme, la question de l'estuaire et du changement climatique étant devenue une thématique centrale en 2016 pour la révision du SAGE ${ }^{2}$.

\section{L'estuaire de la Loire, laboratoire de la transition}

La complexité et la richesse des sujets environnementaux qui caractérisent ce territoire justifient pleinement la place majeure de la thématique de la transition dans le nouveau SCoT Nantes SaintNazaire qui considère l'estuaire de la Loire comme « un laboratoire de la transition énergétique et écologique ». Les objectifs environnementaux développés dans le SCoT se confrontent cependant à la question du fonctionnement de l'espace économique et le développement de l'emploi. Par exemple, le développement ou le maintien des industries implantées sur l'estuaire et est en grande partie orientées vers le secteur énergétique, représentent des symboles du changement climatique par leur contribution aux émissions de gaz à effet de serre (centrale à

\footnotetext{
2. Le 30 juin 2016 les $6^{e}$ Rendez-vous du SAGE ont porté sur les impacts du réchauffement. Cécile Fourmarier, directrice du Syloa et animatrice du SAGE Estuaire de la Loire, a abordé le changement climatique comme le fil conducteur de la révision du SAGE [http://www.sage-estuaire-loire. org/actualites/529-des-solutions-face-au-changement-climatique.html].
} 
charbon de Cordemais, raffinerie de Donges). Ce développement économique nécessiterait aussi la mise en place de projets d'infrastructures impactant l'environnement, tel que le projet d'un nouveau pont entre Saint-Nazaire et Nantes ou encore celui du projet d'aéroport de Notre-Dame-des-Landes situé à proximité du site d'étude (Barbe, 2016). Le projet d'aéroport représente d'ailleurs un argument utilisé par les élus des territoires situés au sud de l'estuaire pour justifier le besoin d'un nouveau franchissement de la Loire permettant un accès plus direct au futur aéroport ${ }^{3}$ illustrant les effets environnementaux indirects de telles infrastructures souvent peu pris en considération ${ }^{4}$. Inversement des projets de protection de l'environnement peuvent aussi représenter un frein au développement de certaines activités. Le projet de réserve naturelle nationale de l'estuaire de la Loire relancé en juillet 2016 par la ministre de l'Environnement Ségolène Royale a provoqué une levée de boucliers de la part d'un collectif, "Collectif 44 des Racines et des Hommes », inquiet des effets de ce projet sur des activités telles que la chasse. Ce projet est aussi pressenti par plusieurs acteurs locaux comme une potentielle « compensation écologique » du projet d'aéroport...

Ces projets, qui représentent des sujets de controverse important au sein de la population locale et nationale, pointent les potentielles incompatibilités entre les actions en faveur de l'économie de l'emploi et celle en faveur de l'environnement, de l'agriculture et du climat. Ils révèlent la complexité qui résulte des interrelations existantes entre les activités et les projets sur un territoire. Les conflits environnementaux contribuent à la construction d'une identité collective, avec la production d'argumentaires et de connaissances expertes nécessaires à la défense des intérêts défendus (Dechézelles et Olive, 2016). Ils peuvent contribuer à une plus grande implication citoyenne et politique des habitants d'un territoire qui peut représenter un vecteur de transition.

L'estuaire de la Loire et plus particulièrement la rive Nord de l'estuaire s'avère donc être non seule-

\footnotetext{
3. Yves Auvinet, le président du Conseil départemental de Vendée rappelle qu'en cas de construction de ce nouvel aéroport « un nouveau pont devrait être construit sur la Loire » afin de faciliter l'accès à l'aéroport pour les Vendéens. Article de presse Le Reporter sablais 20 février 2016 [www. lereportersablais.com].

4. Voir extrait du courrier de Loïc Marion, à Claude Chéreau, Président de la Commission du Dialogue [naturalistesenlutte.wordpress.com/2013/03/20/ le-projet-d-aeroport-menace-aussi-la-reserve-naturelle-degrand-lieu-e/].
}

ment un territoire opportun pour s'interroger sur la mise en œuvre du développement durable (Després, 2009) mais aussi un espace privilégié pour explorer les transitions en cours vers un urbanisme et un aménagement des territoires réduisant les impacts anthropiques sur le climat et minimisant les impacts des changements climatiques sur les territoires.

\section{Remerciements}

Ce travail a été financé par le ministère de l'Écologie, du Développement durable, des Transports et du Logement, dans le cadre du programme de recherche "Conséquence des changements climatiques sur l'écogéomorphologie des estuaires » (C3E2, 2011-2014) du Programme Gestion et Impacts du Changement Climatique et par la Fondation de France dans le cadre du programme de recherche ICEPEL «Impacts socio-économiques des changements environnementaux des complexes prairiaux de l'estuaire de la Loire : approche prospective » (ICEPEL 2015-2017), appel d'offres "Quels littoraux pour demain? » Il a bénéficié de la relecture et des conseils de deux relecteurs anonymes pour la revue Norois.

\section{Bibliographie}

Barbe F., 2016. La «zone à défendre » de Notre-Dame-desLandes ou l'habiter comme politique, Norois, $\mathrm{n}^{\circ} 238-239$, p. $109-130$.

Cabanne C., 1985. Nantes-Saint-Nazaire vers le large, Norois, $n^{\circ} 126$, p. 269-272.

Chambre d'agriculture de Loire Atlantique, GiP Loire estuaire ET DDTM 44, 2013, Caractérisation des exploitations agricoles de Nantes à Saint-Nazaire, Rapport final, mars 2013, $80 \mathrm{p}$.

Cianfaglione K., Bioret F., 2017. Autoecological and Synecological Resilience of Angelica heterocarpa M.J. Lloyd, observed in the Loire Estuary (France), in Greller A., Fujiwara K., Pedrotti F. (eds.), Geographical changes in vegetation and plant functional types, Geobotany studies, Springer, 318 p.

CSEEL, 1984. Rapport final du Comité Scientifique pour l'Environnement de l'Estuaire de la Loire - Rapports Science et Technologie CNEXO, no 55, 158 p.

Day J. W., Christian, R.R., Boesch D.M., Yáñez-Arancibia A., Morris J., Twilley R.R., Naylor L., Schaffner L.et Stevenson C., 2008. Consequences of climate change on the ecogeomorphology of coastal wetlands, Estuaries and Coasts, 31(3), p. 477-491. 
Demaure J.-C., 1979. Les contraintes écologiques à l'aménagement de l'estuaire de la Loire, Penn ar Bed, 97, p. 57-72.

Després L. (Coord.) 2009. L'estuaire de la Loire - Un territoire en développement durable?, Rennes, PUR, coll. « Espace et Territoires », $472 \mathrm{p}$.

Dechézelles S., Olive M. (Coord.), 2016. Conflits de lieux, lieux de conflits, Norois, $\mathrm{n}^{\circ}$ 238-239. $172 \mathrm{p}$.

Dupont P., Bernard J.-Y., Bioret F., 1983. Étude des associations végétales dans les zones humides de l'estuaire de la Loire, Laboratoire d'Écologie et de Phytogéographie, Université de Nantes, 96 p.

Estuarium, 1997. Livret d'exposition Portes d'èbe et portes de flots, Exposition : Estuaire de Nantes à Saint-Nazaire - histoire d'un port - Musée du château des Ducs de Bretagne, novembre et décembre 1997, 22 p.

Fleury D., 1997. Des îles et des ingénieurs, in Les Cahiers du Conservatoire "Îles de Loire », Conservatoire régional des rives de la Loire et de ses affluents, p. 21-34.

GIEC, 2007. Bilan 2007 des changements climatiques, Contribution des Groupes de travail I, II et III au quatrième Rapport d'évaluation du Groupe d'Experts intergouvernemental sur l'évolution du climat, GIEC, Suisse, 103 p.

GIP Loire Estuaire, 2005. Submersibilité latérale dans l'estuaire, Cahiers indicateurs GIP Loire Estuaire, L1D1, 6 p.

GIP Loire Estuaire, 2006a. La chasse au gibier d'eau, Cahiers indicateurs GIP Loire Estuaire, L3A7, 8 p.

GIP Loire Estuaire, 2006b. Études prospectives aval - Quelle orientation pour l'estuaire, Synthèse du CA du GIP 8 décembre 2006. 20 p.

GIP Loire Estuaire, 2007. Études prospectives aval - Les scénarios, Programme interrégional Loire grandeur nature Pays de la Loire 2000-2006, GIP Loire Estuaire - juin 2007, 16 p.

GIP Loire Estuaire, 2011 a. Les syndicats de marais, Fiche GIP Loire Estuaire - Les marais estuariens de la Loire, 4 p.

GIP Loire Estuaire, 201 lb. Les dragages d'entretien dans l'estuaire, Cahiers indicateurs GIP Loire Estuaire, L1E3, 4 p.

GIP Loire Estuaire, 201 lc. Les prélèvements et rejets d'eau, Cahiers indicateurs GIP Loire Estuaire, L3A2, 8 p.

GIP Loire Estuaire, 2012. Submersibilité latérale dans l'estwaire, Cahiers indicateurs GIP Loire Estuaire, L1D1, 8 p.

Gourmelon F., Bioret F., Rebout C., Yésou P., 2003. Les dynamiques de la végétation d'un îlot marin protégé, Photo Interprétation, 39 (2), p. 3-13.

Gras J., 1981. L'estuaire de la Loire : une étude générale d'environnement, Norois, 109, p. 5-30.

Guillemot B., 2015. Enjeux et pistes d'actions pour la sauvegarde des équilibres fondamentaux de la Loire. Synthèse de l'intervention « Le grand débat - Nantes, La Loire et nous La Loire, espace économique, espace écologique », Nantes Métropole. 4 p.

Hill M.O., Mountford J.O., Roy D.B., Bunce R.G.H., 1999. Ellenberg's indicator values for British plants, vol. 2, ECOFACT, CEH, UK, ISBN 1870393481, 46 p.

Hobbs R. J., Higgs, E.J., Harris A., 2009. Novel ecosystems: implications for conservation and restoration, Trends in Ecology et Evolution 24, p. 599-605.
INSEE, 2008. L'impact socio-économique du Port de Nantes Saint-Nazaire, Direction régionale de l'INSEE des Pays de la Loire - Université de Nantes, 31, 28 p.

INSEE, 2011 . Unités urbaines de plus de 100000 habitants en 2011, Institut national de la statistique et des études économiques, [http://www.insee.fr].

Le Dez M., Sawtschuk J., Bioret F., 2017. Les prairies de l'estuaire de la Loire : étude de la dynamique de la végétation de 1982 à 2014, Mappemonde, 119,18 p. [http:// mappemonde.mgm.fr/119as2/?pdf=1530].

Le Hir P. (coord.), 2014. Conséquences du Changement Climatique sur l'Ecogéomorphologie des Estuaires, Programme Gestion et Impacts du Changement Climatique (GICC), rapport final, partie 2, $160 \mathrm{p}$.

Leiderman E., Mermet L., 1991. Mise en place d'un observatoire de zones humides. Identification de zones humides d'importance majeure au plan national, Ministère de l'Environnement - DNP, AIFA et SPRN (non-publié).

Magnanon S., Dupont P., Bioret F., 1998. Angelica heterocarpa dans l'estuaire de la Loire : répartition, écologie, menaces. Propositions de mesures de gestion, Conservatoire Botanique National de Brest, DIREN Pays de la Loire 25 p. + cartes et annexes.

Mareï N., Tourret P., 2009. Les industries maritimes, les territoires et leur organisation, Cahiers Nantais, 1, p. 5-17.

Mauvais J. L., Goarnisson R., 1999. État de l'environnement sur la façade atlantique, Paris, Quae, 140 p.

Migniot C., Le Hir P., et Association pour la protection de l'environnement de l'estuaire de la Loire. 1997. Estuaire de la Loire, rapports de synthèse de l'APEEL 1984-1994, Nantes, APEEL, Association pour la protection de l'environnement de l'estuaire de la Loire, 103 p.

Moatar F., Vidal J.-P., Billen G., 2010. Présentation du projet ICC HYDROQUAL : Impact du changement climatique sur l'hydrosystème Loire: HYDROlogie, régime thermique, QUALité [http://www.plan-loire.fr/fileadmin/pce/PFRDI/ Reunions/Docs/Presentations/presentationICCHydroqual090210.pdf].

Oтtмan F., 1987. Les modifications physiques de l'estuaire de la Loire et leurs conséquences socioéconomiques, Norois, no 133-135, p. 81-89.

Ouest Aménagement, 2003. Inventaire et cartographie Natura 2000 de la Loire en aval de Nantes - volume 1 : flore et végétation, DREAL Bretagne, 80 p. + cartographies.

Robins P.E., Skov M.W., Lewis M.J., Giménez L., Davies A.G., Malham S.K., Neill S.P., McDonald J.E., WhitTON T.A., Jackson S.E., JaGo C.F., 2016. Impact of climate change on UK estuaries: A review of past trends and potential projections, Estuarine, Coastal and Shelf Science, 169, p. 119-135.

SAEIJS H.L.F., 1982. Changing estuaries. A review and new strategy for management and design in coastal engineering, Rijkswaterstaaat Communications, 414 p.

Sawtschuk J., Bionet F., 2012. Analyse diachronique de la dynamique spatiale de la végétation de l'estuaire de la Loire. Photo-interprétation, $\mathrm{n}^{\circ}$ 48, p. 15-28. 
Usher M.B., 1992. Statistical models of succession, in GLENNLewin D.C., Peet R.K., Veblen T.T. (eds.) Plant succession: theory and prediction, London, Chapman et Hall, p. 215248.

Verger F., 2005. Marais et estuaires du littoral français, Paris, Belin, 335 p.

Walther R., David E., 2010. 3D Modeling of Salinity and Sediment Suspension in the Loire Estuary: Coupling of
Processes, in Practical Applications in Engineering (Environmental Hydraulics) (ed J.-M. Tanguy), vol. 4, Hoboken, NJ, John Wiley et Sons, Inc., 424 p.

Walther R., Schaguene J., Hamm L., David E., 2012. Coupled 3D modeling of turbidity maximum dynamics in the Loire estuary, Coastal Engineering Proceedings, 1(33), 11 p. [http://dx.doi.org/10.9753/icce.v33.sediment.22]. 